\title{
Interventional Therapy of Cerebrovascular Disease Mechanical
}

\section{Embolic Materials}

\author{
Qiang Sun ${ }^{1}$, Jiang Yuan ${ }^{1}$, Lei Zeng ${ }^{2, a^{*}}$ \\ ${ }^{1}$ Department of Neurology, Taihe Hospital, Hubei University of Medicine, Hubei, China \\ ${ }^{2}$ Department of Radiology, Ren-Min Hospital, Hubei University of Medicine, Hubei, China \\ *Corresponding author: Lei Zeng, E-mail:sq2015istp@163.com
}

Keywords: interventional therapy; cerebrovascular disease; embolic materials

\begin{abstract}
Intravascular embolism has become the major means for treating intracranial aneurysm, cerebral arteriovenous malformation and arteriovenous fistula. With the development of mechanical embolic materials, especially mechanical detachable tungsten filament micro spiral, guglielmi datacbcble micro coil and other controlled micro spiral techniques, intracranial aneurysms can almost achieve the same effect of surgical clipping. However, all kinds of mechanical embolic materials have their advantages and disadvantages in clinical application. In order to meet the needs of different parts, different nature of the disease, the development and application of new materials in the endovascular treatment field has been carried out. The mechanical embolic materials are of two types: micro spirals and detachable balloon. The report briefly introduces the types and efficacy of mechanical embolization materials.
\end{abstract}

\section{Introduction}

Interventional neuroradiology is also called endovascular neurosurgery which injects some artificial embolism materials manipulatively into the supplying blood vessels or lesion vessels of lesion organs so as to make it block and interrupt the blood supply for the purpose of controlling bleeding, curing vascular diseases, tumors and eliminating the lesion organ functions. Its development has brought about good news for patients suffering brain diseases. The ideal embolism materials should meet below requirements: non-toxic, no antigenicity, good biocompatibility, can rapidly block blood vessels, can stop up blood vessels with different diameters and flows, easy for catheters to convey, easy to be disinfected, and can control the time duration to block blood vessels, and can achieve percutaneous recycling or revascularization once needed. This article has made a general summary on current frequently-used mechanical embolism materials in the neurointervention treatment.

1. Micro Spirals Its Good biocompatibility, chemical stability, non-toxic, easy to through the duct system, in the tumor cavity induce thrombosis, is currently the clinical blood tube embolism materials [1] for the treatment of intracranial aneurysm is the most commonly used. Suitable for traumatic carotid cavernous sinus fistula, intracranial aneurysm and arteriovenous fistula.

1.1 Guglielmi Datacbcble Coil (GDC) Micro thruster with platinum spring coil connection using micro welding technology. GDC is composed of three parts: the recent $(175 \mathrm{~cm})$ made by stainless steel metal core, middle part made by the very soft stainless steel ring, the terminal is made of a platinum coil. GDC - 10 and GDC - 18 two kinds of models, each model and three kinds of specifications, the single diameter, dual diameter, $3 \mathrm{~d}$ model. Diameter of single and dual diameter 
is divided into standard type and soft. Standard to form the top bracket, aneurysm filling type soft filling the space between the top bracket, to achieve the goal of closely tamponade aneurysms. Three dimensional model for coil forming within the aneurysm, the micro spring can contact with the inner wall of the aneurysm is very good, the spring coil from from tumor neck out, block parent artery. Recently introduced resistant solutions for helical spring coil, the need to adjust the position of the coil inside the aneurysm don't have to send back and forth in a spiral straightened, exit make spring coil and cannot be used. Advantages: the spring coil is very soft, within the micro tube in a straight line, without resistance, can be suitable aneurysm shape and size and shape, even in the form of $2 \mathrm{~d}$ or $3 \mathrm{~d}$ in the body cavity tumor assumes the top structure, not easy from the aneurysm cavity of emergence, compact tamponade in aneurysm cavity, and the tumor wall is only slight pressure. Fully controllable spring coil. Its micro propeller with platinum spring coil with micro welding technology to connect, operation can adjust the position of the spring coil repeatedly until appropriate electrolytic relief, is by far the most ideal embolization combined with the material, can be directly generated by electric coagulation blood clots, can be completely filling the aneurysm, curative effect is satisfactory [2]. Disadvantages: manufacturing complex and expensive.

1.2 EDC It is a kind of electric relief type spring coil, similar to GDC, but its push the head end of the guide wire is soft spring, spring coil after liberation, the broken end is not easy to damage to the artery wall, join fusing faster. Dendron company new multi-point release spring coil (variable detachable system, VDS), in a micro spring have more than one release point, fill without replacement can be realized with the length of the optional sex, may make the aneurysm filling is more convenient and effective [3] .

1.3 Machenical Detachable Tungsten Filament Micro Spiral(MDS-N) Its performance and effect similar to GDC, it is the main difference between tungsten wire coil in mechanical way liberation, can free back or reposition the coil and position until satisfied, beyond the propeller tip catheter, coil can be immediately released from tumor cavity. Disadvantages: incomplete embolization, thrombus formation rate is low, intraoperative rupture hemorrhage, complex and expensive, manufacturing process, is not an ideal embolic material. Mainly used in the treatment of intracranial aneurysm. MDS using mechanical method relief tungsten coil, beyond the propeller tip catheter, spring coil can be immediately released from tumor cavity, controllability, less than GDC [4].

1.4 Spiral Detachable Micro Spiral(DCS) It made of platinum materials, pre-installed on the controlled release system. When DCS completely, as it enters the aneurysm in micro guide tubes counterclockwise (CCW) rotation from locking system (20 25 times, DLD clutches) in 30 seconds from DCS. It has the following types: (1) spiral: mainly used for playing hemangioma and larger than $2.5 \mathrm{~cm}$ in diameter of AVF embolism. (2) the head end more spiral J shape (MCJ) : for 3, 4, a screw head end, straight back into shape. For it needs a series of the circumstances as the first embolus embolus, also suitable for continuous use. The spiral favorable embolus in the tumor cavity is fixed, the straight part of the free to look for space, padded along the wall of tumor. (3) J shape: the head end for a spiral, straight back into shape. Standard form is only suitable for giant aneurysms, soft shape is suitable for large and small aneurysms. (4) shape: suitable for small curved artery embolism. (5) tower: from coarse to fine spiral structure, suitable for short and anatomy of embolism, in the use effect of aneurysm neck very well [2]. Compared with GDC: (1) the installation is simple; (2) without electricity; (3) release faster; (4) the price is cheap, but owe all 
specifications, need to manually release, if push godet have crease or blood vessels are too curved, rotating release that may will micro catheter pop-up, need to be careful operation. In addition, if push godet beyond micro tube too much, the required number of turns is released will increase, and even can't liberation. As a result, the signs on the micro catheter and push the signs on the thread should be properly involution .

1.5 Detachable hydrolysis spiral The main features are: (1) soft, filling type platinum ring softness and soft type GDC - 10. (2) consistency, good adherent force is small. (3) centripetal filling, forming a natural appeared, from outside to inside of centripetal distribution characteristics, stuffing fully (comparable to similar products more than 39\%); Gently stick wall, filling and rarely appeared in the process of relief micro catheter tip to swing, the springback. (4) water escape: no biochemical reactions and possible for electrical stimulation by bubbles and thrombosis. Release quickly (the average platinum ring free time for 1-3 seconds), reliable, free since going public in 2000, there is no failure report. (5) form a complete set of prefabricated forming micro catheter, is easier to reach the lesion location, and strengthen the support and meet the needs of operation for a long time.

1.6 FGF - Core Spring Coil A new type of platinum micro spring coil, it is a used to absorb and release of endovascular treatment for intracranial aneurysm pva core of active material. Method is a short period of PVA line embedded at the center of the original spring coil, the coil is called PVA core spring coil. The PVA - core spring coil into promoting the fibroblast growth factor (bFGF) in the solution for an hour. Let the PVA core absorption bFGF made FGF - core spring coil. In vitro experiments show that PVA - core within 24 hours will be released into the salt solution bFGF. Animal experiments showed that the 14 and 28 days, FGF - core spring coil into group rat carotid artery lumen, have significant cell reproduction; Hyperplasia of cell components around the implanted coil diffusion, the ingredients most be Ma Sensan color dyed blue. These changes in the implantation of PVA - core spring coil and unprocessed spring coil group did not occur. So the researchers think the FGF - core spring coil induced fibrosis change is effective in the aneurysm. The spring coil can be used for treatment of cerebral aneurysm embolization material [5]. The spring coil is not yet in clinical application, its effect remains to be proven.

1.7 Copper Embolizing Agent Copper good to plug, copper tungsten ring with the physical and chemical properties, according to a study by copper coated tungsten ring do not change the memory of tungsten ring, bending resistance and strength, in conveying experiments, experimental study, and no obvious difference was found between tungsten ring, but the bolt sex is better than tungsten ring. As an active metal, with the strong blood clot caused by present fast, easy to shape, don't break down in the body, conveniently, the advantages of cheap, is a kind of ideal embolic agents material. But copper is safe and stable long-term indwelling the body remains to be further in-depth study. Recent strong corrosion resistance, high stability of copper to copper memory metal embolization agent development provides new materials.

2. Detachable Balloon Two kinds of latex balloon and silica gel. With the balloon filled with permanent fillers, and micro catheter with use, until after the balloon into the tumor and inflation, then gently pulling catheter can free balloon. Inside and outside the country in the past, the treatment of aneurysms and the detachable balloon embolization of the aneurysm cavity, but there are defects are as follows: (1) balloon set bit technology is more complex; (2) balloon must be filled with a permanent embolic agent (HEMA) in order to maintain the permanent blocking; (3) balloon 
filling degree is difficult to grasp, even supporting the risk of rupture of aneurysm; endovascular embolization after the water hammer effect, resulting in recurrence of aneurysm or rupture, more complications, mortality high . At present, this method has been largely abandoned [6]. At present, only suitable for the treatment of basilar artery aneurysms, ophthalmic aneurysms, occlusion and carotid cavernous fistula in the basilar artery. First reported by the Moret [7] balloon assisted treatment of wide necked intracranial aneurysms embolization, known as the parent artery "remodeling" technology . Preliminary report on treatment of 15 cases of intracranial aneurysms with wide neck, satisfactory results were reported.

\section{Conclusion}

The neuro-interventional embolization treatment is applicable for cerebral aneurysm, carotid artery cavernous sinus fistula, vertebra arterio-venous fistula, carotid artery and vertebral artery aneurysm, Intradural perimedullary arteriovenous fistulae, spinal dural arteriovenous fistula, and spinal canal vein pressure, etc. The correct selection of embolism materials should be based on acquaintance with its characteristics, the required blocking level, blocking time, and potential complications. GDC and MDS can be used for intracranial aneurysm greatly enhancing the security of embolism intracranial artery. The embolism indication of arterial aneurysm is getting wider and wider. Especially for posterior circulation arterial aneurysm or large-scale arterial aneurysm which is hard to get access and occlusion by operations, embolism arterial aneurysm can be applied to achieve the treatment goal. Althouth GDC and MDS has good curative effect on diseases such as intracranial aneurysm,there still remain problems such as the imcompletion of embolism,low rate of thrombogenesis, intranperative rupture hemorrhage,high costs and complicated manufacturing process, etc.

\section{Reference}

[1] Picard L., Bracard S., Lehericy S: Endovascular occlusion of intracranial aneurysms of the posterior circulation: comparison of balloons, free coils and detachable coils in 38 patients. Neuroradiology, 1996 , 38(1) : 133-141.

[2] Raymond J., Roy D., Bojanowski M: Endovascular treatment of acutely ruptured and unruptured aneurysms of the basilar bifurcation. J Neurosurg, 1997 , 86 (2) : 211-219.

[3] Henkes H., Drepper P., Speder J., Hill J.: Technical note on VDS: a ystemfor endovascular electrolytical detachment of platinum coils at variable length. Interven Neuroradiol, 2002, 8: 197.

[4] Lanzino G., Wakhloo A.K., Fessler R.D.: Efficacy and current limitation of intravascular stents for intracranial internal carotid, vertebral, and basilar artery aneurysms. J Neurosurg, 1999, 91 (4): 538.

[5] Matsumoto M., Hiroyuki W.K., Terada, T.M.: Basic fibroblast growth factor released from a platinum coil with a polyvinyl alcohol core enhances cellular proliferation and vascular wall 
thickness: An in vitro and in vivo study. Neurosurgery, 2003, 53 (2): 402-408.

[6] Byrne J.V., Sohn M.J., Molyneux A.J.: Five-year experience in using coil embolization for ruptured intracranial aneurysms : outcomes and incidence of late rebleeding. J Neurosurg, 1999, 90(4): 656.

[7] Murayama Y.F., Vinuela F.W., Ulhoa A.W: Nonadhesive liquid embolic agnet for cerebral arteriovenous malformations: preliminary histopathological studies in swine rete mirabile. Neurosurgery, 1998, 43(5): 1164. 